\title{
A Study on Local Variance Threshold Based Boundary Detector with Median Filter
}

\author{
Dr. D. Regan ${ }^{1}$, Dr. M. V. Raghavendra ${ }^{2 *}$, Dr. R. Purushotham Naik ${ }^{3}$ \\ 1, 2, 3 Associate Professor, Avanthi College of Engineering and Technology, Hyderabad, India.
}

*Corresponding Author: M. V. Raghavendra, Associate Professor, Avanthi College of Engineering and Technology, Hyderabad, India.

\begin{abstract}
This paper investigates the boundary detection algorithm based on local variance threshold technique. Boundary detection, contour detection, and edge detection are the fundamental research problems in computer vision. They are the primary step in image recognition. Image segmentation is made easy when the outer shape of the object is identified clearly. In this paper, the boundary detection is performed with the local variance of the pixels under calculation. The simulation results outperform the standard edge detection methods.
\end{abstract}

Keywords: Edge detector, Local variance, Boundary detection, Local Variance based detection threshold

\section{INTRODUCTION}

Image segmentation and recognition are the primary steps in every industrial automation fields. The use of object boundary detection is becoming prevalent in every automated industry. The object boundary detection enables the system to identify the shape of the object. Human perception system understands the objects based on the outer shape only. Besides many techniques are proposed in segmentation, outline-based methods would be commonly followed and preferred. Object boundary can be known as a collection of pixels in the image perceived by human visual system [13]. The system proposed in [2] explains the boundary detection of pixels using the local brightness, color and texture cues. The author [1] proposed the edge detection based on the local variance of the pixels in the image.

Contour or boundary of the object can be accomplished in the three methods; the region based, edge oriented and hybrid of these methods [13]. Several edge detection algorithms proposed in [5, 6, 8]. All these methods implemented in use of the directional gradient values of the image after filtering. In the edge detection procedure, thresholding is a vital step. The edges are formed due to the abrupt changes in the intensity value [3]. But not all the edges formed by the sudden changes in intensity values, gradual changes also form the edges. So the objective of the boundary detection would be achieved well if the edges of the object found. The various edge detection methods and comparison are presented in [11].

The Sobel, Robert's cross, Prewitt, and Laplacian of Gaussian (LoG] are the edge detection algorithms based on gradient calculation [10]. The gradient method involves in the detection of the edges by searching for the maximum and minimum in the first derivative of the pixel intensity value of the image. These edge detectors use special vertical and horizontal convolution kernels to detect the gradient changes in these directions.

\section{CAnNy Edge Detection Algorithm}

Moreover among the different edge detection concepts, still the canny edge detection algorithm is the optimal and standard detector. Its response to the edge and non-edge pixel is superior, the localization error of exact edge point is very minimum and only one edge point is identified in edge detection of the pixel. In accordance with these criteria, canny edge detector performs the following steps for edge detection. 


\section{Step1:}

Before going to edge detection, the noises in the image should be removed. For this purpose, the Gaussian smoothing filter is used. The Gaussian filter with different convolution mask is used to reduce noise. The sensitivity to noise and error in the localization of edge points would be avoided because of the Gaussian smoothing filtering process. The convolution kernel mask size is proportional to the error in localization error, so the preferable kernel is small.

\section{Step2:}

After the elimination of noises in the image, the smoothed image is filtered with the Sobel mask. The vertical and horizontal kernel of the Sobel mask slide over the image to detect the gradient of the pixel points in the $\mathrm{X}$ and $\mathrm{y}$ directions. The magnitude of the gradient is calculated to estimate the strength of the edge.

\section{Step3:}

From the gradient of the image in $\mathrm{X}$ and $\mathrm{y}$-direction, the direction of the edges determined.

\section{Step4:}

After the edge directions are calculated, non-maximum suppression has to be followed. Nonmaximum suppression is used to trace along the edge points in the edge direction and suppress any pixel value (sets it equal to 0 ) that is not considered to be an edge.

\section{Step5:}

Eventually, hysteresis is used as a means of eliminating streaking. Streaking is the breaking up of an edge contour caused by the operator output fluctuating above and below the threshold, and then edge linking performed to have a line of edges in the output.

\section{ThreShOLding BaSED MethodS}

There have been numerous survey literatures on thresholding techniques. The outline of the survey conducted in [9] presents six methods of thresholding methods. They are histogram based, clustering based, entropy based, the object attribute based, the spatial methods and the local methods. In the local methods, the local statistics of pixels like range, variance and contrast could be used to estimate the edge location.

\section{LOCAL VARIANCE BASED ALGORITHM}

The local variance of image-based technique proposed [7] for angiogram edge detection. The local variance technique is entirely depending on the statistical nature of the pixels in the image. The value of local variance changes across the edges on comparing to the flat region of the image. Across the edge, the local variance changes from minimum to maximum and maximum to minimum. The local variance of the region is different [7] compared to the region of having edges. This difference between region can be utilized in edge detection [1]. The local variance of the pixel can be computed as given below:

$I_{\text {var }}(X)=E\left(X^{2}\right)-E(X)^{2}$.

$I_{\text {var }}(X)$ - Variance of the pixel

$E(X)^{2}-$ Mean of the pixel

In local variance based thresholding method, the mean of the variance of the pixels under the mask is calculated [5]. The pixel intensity of a number of pixels chosen each time is used in the image region for variance calculation. The mean of the variance of the pixels determines the threshold level to the pixel classification whether the pixel is edge pixel or non-edge pixel.

\section{SYSTEM MODEL FOR BOUNDARY DETECTION}

In this proposed work, firstly the input image is added to the salt and pepper noise. The noisy image is filtered with the median filter. The median filter is commonly used in salt and pepper noise [10], Gaussian noise removal during image denoising, as it preserves the edge strength mostly. There are many median filter algorithms present to treat the denoising problems. For the efficient and edge- 
preserving performance, the median filter would be a preferable tool [12]. Secondly, the noise filtered image is undergone local variance based thresholding to yield the segmented image. The boundary of the objects in the image is obtained by the mean of the local variance of pixels.
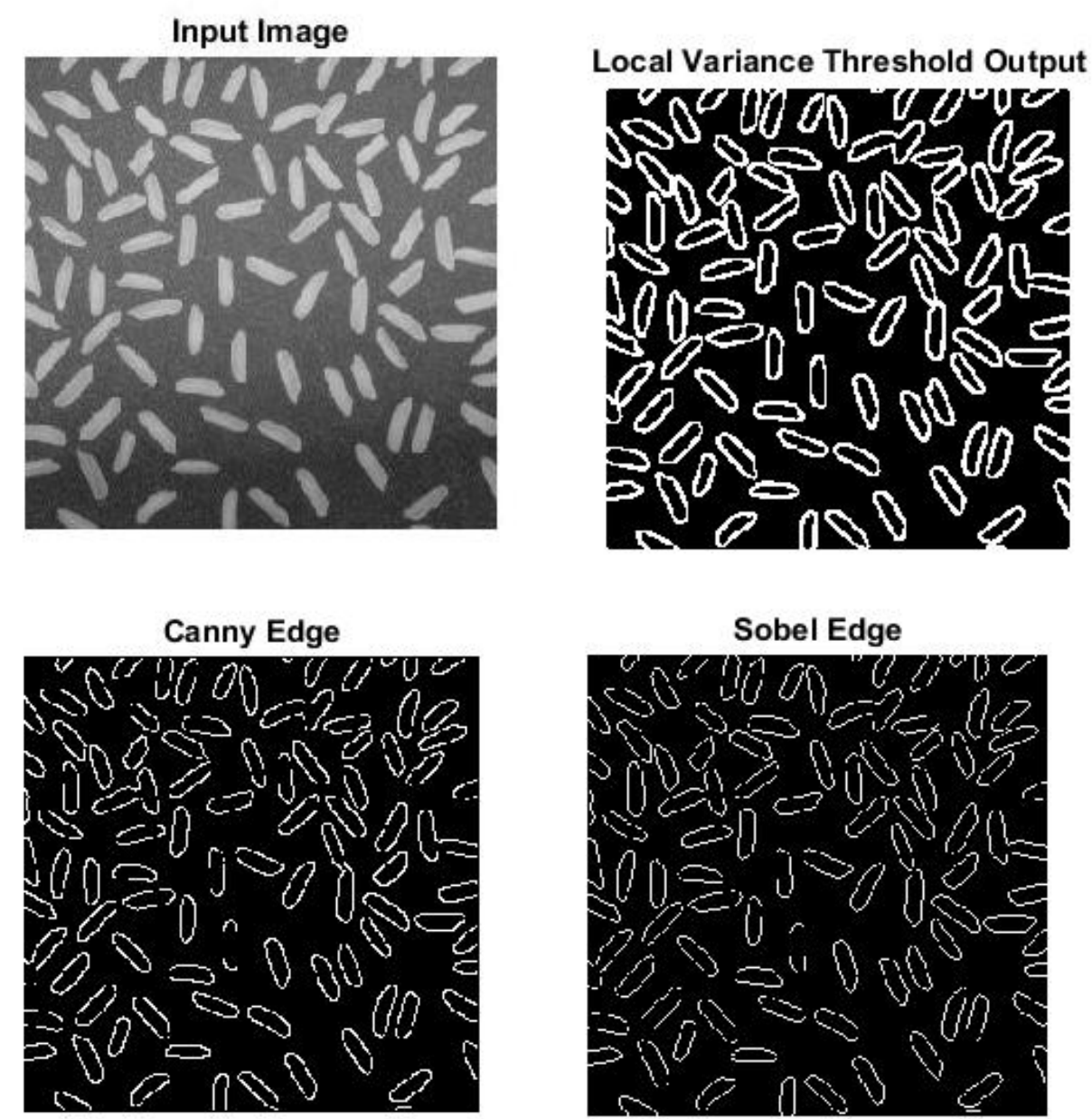

\section{RESULTS}

In this paper, the local variance based object detector is used to detect the boundary of the objects in the image under denoised filtering. The performance of the proposed system is compared with the standard canny and Sobel edge detectors. The visual comparison shows that the local variance based thresholding method outperforms the canny and Sobel edge detectors.

\section{REFERENCES}

[1] A. Fabijańska, "Variance filter for edge detection and edge-based image segmentation," Perspective Technologies and Methods in MEMS Design, Polyana, 2011, pp. 151-154.

[2] D. R. Martin, C. C. Fowlkes and J. Malik, "Learning to detect natural image boundaries using local brightness, color, and texture cues," in IEEE Transactions on Pattern Analysis and Machine Intelligence, vol. 26, no. 5, pp. 530-549, May 2004.

[3] E. Argyle and A. Rosenfeld, "Techniques for edge detection," in Proceedings of the IEEE, vol. 59, no. 2, pp. 285-287, Feb. 1971.

[4] https://angeljohnsy.blogspot.com

[5] J. Canny, "A Computational Approach to Edge Detection," in IEEE Transactions on Pattern Analysis and Machine Intelligence, vol. PAMI-8, no. 6, pp. 679-698, Nov. 1986.

[6] J. M. S. Prewitt, “Object enhancement and extraction," Picture Processing and Psychopictorics, B. Lipkin and A. Rosenfeld, Eds., New York: Academic Press, 1970, pp. 75-149.

[7] Liu, X., Chen, S., Zou, M. et al. J. of Electron.(China) (2000) 17: 338. https://doi.org/10.1007/s11767-0000008-X.

[8] M. Petrou and J. Kittler, "Optimal edge detectors for ramp edges," in IEEE Transactions on Pattern Analysis and Machine Intelligence, vol. 13, no. 5, pp. 483-491, May 1991. 
[9] Mehmet Sezgin, Bülent Sankur, "Survey over image thresholding techniques and quantitative performance evaluation," J. Electron. Imag. 13(1) (1 January 2004).

[10] Gonzalez, R. C., \& Woods, R. E. 1. (2008). Digital image processing (3rd ed.). Upper Saddle River, N.J.: Prentice Hall.

[11] Raman, Maini \& Aggarwal, Himanshu. (2009). Study and Comparison of Various Image Edge Detection Techniques. International Journal of Image Processing. 3.

[12] S. Khatri and H. Kasturiwale, "Quality assessment of Median filtering techniques for impulse noise removal from digital images," 2016 3rd International Conference on Advanced Computing and Communication Systems (ICACCS), Coimbatore, 2016, pp. 1-4.

[13] Y. Ming, H. Li and X. He, "Connected contours: A new contour completion model that respects the closure effect," 2012 IEEE Conference on Computer Vision and Pattern Recognition, Providence, RI, 2012, pp. 829-836.

\section{AUTHORS' BIOGRAPHY}

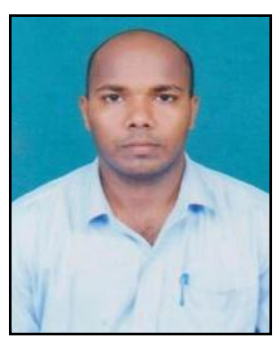

Dr. D. Regan, is currently working with Avanthi Institute of Engineering \& Technology, Hyderabad in dept. of ECE as Associate Professor. He has 10 years of academic experience with 1 year in ISRO as Graduate Apprentice Trainee. He received his PhD from St.Peter's University, Chennai, India in Remotely sensed image processing, and got his M.Tech in Applied Electronics from Dr.MGR University, Chennai, India. Also he graduated B.E (ECE) in 2004 from University of Madras. He published 9 research papers in national and international journals. His Research interest is multispectral and hyperspectral image processing, digital

image processing.

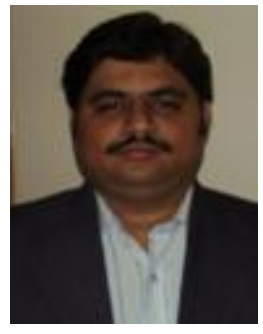

Dr. Venkata Raghavendra Miriampally, is currently working as Professor, in Electronics and Communication Engineering Department, Avanthi Institute of Engineering \& Technology, Hyderabad. He has worked in Electrical Engineering Department, Adama Science \& Technology University, Ethiopia. He received his $\mathrm{PhD}$ from Singhania University, Rajasthan, India in the field of Optical Communication, and he has received his M.Tech (radar \& Microwaves) Degree from ECE Dept., College of Engineering, Andhra University, Vishakhapatnam, India. His main research includes signal estimation \& evaluation of optical communication, Satellite communication, Microwaves \& Radar. He has published papers in reputed national \& international Journals. He has participated in different national \& international conferences. He is having life memberships of MISTE, MISOI, MIAENG, MIACSIT, MAIRCC, MSCIEI, SMUACEE, PASS, MISMTE, MMEASCE, and SMIRED.

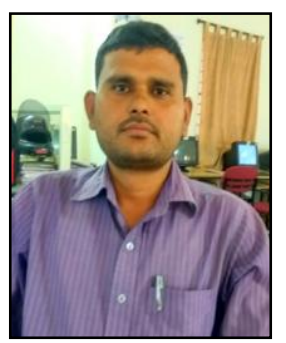

Dr. R. Purushotham Naik, is currently working as a Assoc. professor,in Electronics and Communication Engineering Department, Avanthi Institute of Engineering \&amp; Technology, Hyderabad. He has worked in Electronics and Communication Engineering Department, Princeton College of Engineering \&amp; Technology, Hyderabad. He received his Ph.D from Sunrise University, Rajasthan, India .In the field of Low power VLSI, And he has received his M.Tech (VLSI) in Electronics and Communication Engineering Department, Anurag College of Engineering, Jawaralal Nehru Technology University of Hyderabad, India. His main research includes High Speed Circuit Design of Low Power VLSI (Delay, Power, and Area).He has published papers in national and international journals. He has participated in national and international conferences. He is having life membership of MISTE.

Citation: M. V. Raghavendra et.al. (2018)" A Study on Local Variance Threshold Based Boundary Detector with Median Filter", International Journal of Innovative Research in Electronics and Communications (IJIREC), 5(1), pp.8-11. DOI: http://dx.doi. org/10.20431/2349-4050.0501002

Copyright: (C) 2018 M. V. Raghavendra, This is an open-access article distributed under the terms of the Creative Commons Attribution License, which permits unrestricted use, distribution, and reproduction in any medium, provided the original author and source are credited. 